\title{
Perspective
}

\section{Using a breathing simulator to improve simulation-based education for noninvasive ventilation}

Noninvasive ventilation (NIV) is a well-recognised management strategy for acute hypercapnic respiratory failure. Lack of appropriate training can reduce the efficacy of this therapy, which may impact on patient safety. Simulation is already used as a training tool in the management of patients requiring NIV. Novel technologies such as breathing simulators have the potential to revolutionise how we train healthcare professionals to manage patients who require treatment with ventilatory support.

Over the past year, the Medisim team at the Royal Surrey Hospital Simulation Centre has explored using the Active Servo Lung (ASL) 5000 breathing simulator (IngMar Medical, Pittsburgh, PA, USA). This breathing simulator allows the manikin to generate airflow through the upper airways. A range of respiratory conditions can be realistically simulated by minutely adjusting the breathing simulator's resistance, compliance and effort settings. Furthermore, the breathing simulator's generated airflow accurately interacts with an attached ventilator. This innovation has enabled us to develop high fidelity NIV-based scenarios, massively enhancing the training delivered. We discuss some of the simulation scenarios we have developed and provide a supplementary video demonstrating how we have made use of this novel technology.

\section{Educational aims}

- To discuss how the use of a breathing simulator can enhance training in NIV.

- To provide practical considerations for setting up and delivering an optimal NIV simulation scenario using this novel technology.

\section{Background}

Acute hypercapnic respiratory failure frequently necessitates the application and use of NIV. NIV is a potentially life-saving therapy, which refers to the provision of respiratory support to the patient's upper airways using a well-fitting mask and a specialist ventilator. Lack of appropriate training has been shown to reduce the efficacy of NIV in the management of acute respiratory failure [1].

There is now good quality research evidence demonstrating that simulation-based education is highly effective and may result in improved patient care and clinical outcomes [2]. Simulation-based medical education is already used as a training tool in the management of patients requiring NIV [3]. However, the technical capabilities of any simulation manikin used may limit the efficacy of training and hinder learning opportunities.
Cite as: Turner D, Picton G Harrod E, et al. Using a breathing simulator to improve simulation-based education for noninvasive ventilation. Breathe 2021; 17: 200285.

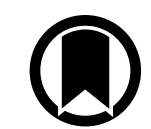

CrossMark 
Novel technologies such as breathing simulators have the potential to revolutionise how we train healthcare professionals to manage patients requiring NIV. Breathing simulators can realistically replicate a vast number of disease states, which can be used for educational purposes as well as the testing of ventilators and other respiratory equipment. Realism, or how close the simulation is to reality, is a highly important concept in simulationbased medical education. A true perception of realism by the learner maximises interaction, engagement, motivation and learning [4]. Hyperrealistic, immersive simulation may increase candidate stress levels, but effective pre-briefing can mitigate this.

\section{Discussion}

The ASL breathing simulator allows SimMan 36 (Laerdal, Orpington, UK) to "breathe", i.e. generate a flow of air through the upper airways of the manikin (figure 1). If you feel closely over SimMan's mouth, one can physically detect air movement, and this feature can be used to add realism to any simulation scenario.

In contrast to the use of SimMan without the breathing simulator, this technology allows the operator to generate enough airflow to trigger an external ventilator to deliver a support breath to a manikin. It also facilitates the control of the resistance and compliance of the simulated lung in order to control the amount of pressure support required to achieve a set tidal volume. Control of resistance and compliance of the simulated lung is minutely adjustable "on the fly" by the individual remotely operating the ASL 5000 breathing simulator via the Laerdal Learning Application (LLEAP) computer platform. This allows the instructor to guide the simulation scenario in various desired pathways dependent on the candidate's learning requirements. If the operator of the simulated lung increases the resistance to a high value, set pressure support on the ventilator may become inadequate and tidal volumes will subsequently reduce. The ventilator will detect and display reduced tidal volumes, respiratory support may be insufficient and emergency alarms will be triggered, appropriately alerting the candidates as it would in reality. The operation of the ASL 5000 breathing simulator using the LLEAP platform and the impact of changing lung resistance, effort and compliance is demonstrated in the supplementary video.

We have developed a number of simulated scenarios across our medicine and intensive care simulation programmes using this novel technology. These focus on the initiation, optimisation and escalation of patients who require NIV. These scenarios use a combination of the breathing simulator connected to SimMan 3G and a noninvasive ventilator in current clinical use in our Trust (Phillips V60; Philips Respironics, Eindhoven, the Netherlands).

One such scenario focuses on the initiation of NIV support to a patient. The patient is initially short of breath with a high respiratory rate and an oxygen requirement. This clinical scenario was simulated by setting the breathing simulator to achieve an effort sufficient to trigger ventilator delivered support breaths with resistance set relatively low. Arterial blood gas analysis shows a respiratory acidosis indicative of hypercapnic respiratory failure. The patient continues to deteriorate and become more clinically unwell until the candidate initiates NIV. The candidate will need to consider appropriate mask sizing and fitting, initial ventilator settings, mask adjustment to avoid excessive leak, etc.

Once the patient is successfully established on NIV the scenario progresses and the patient develops a tension pneumothorax on one side. To simulate this complication, the breathing simulator operator rapidly increases the lung
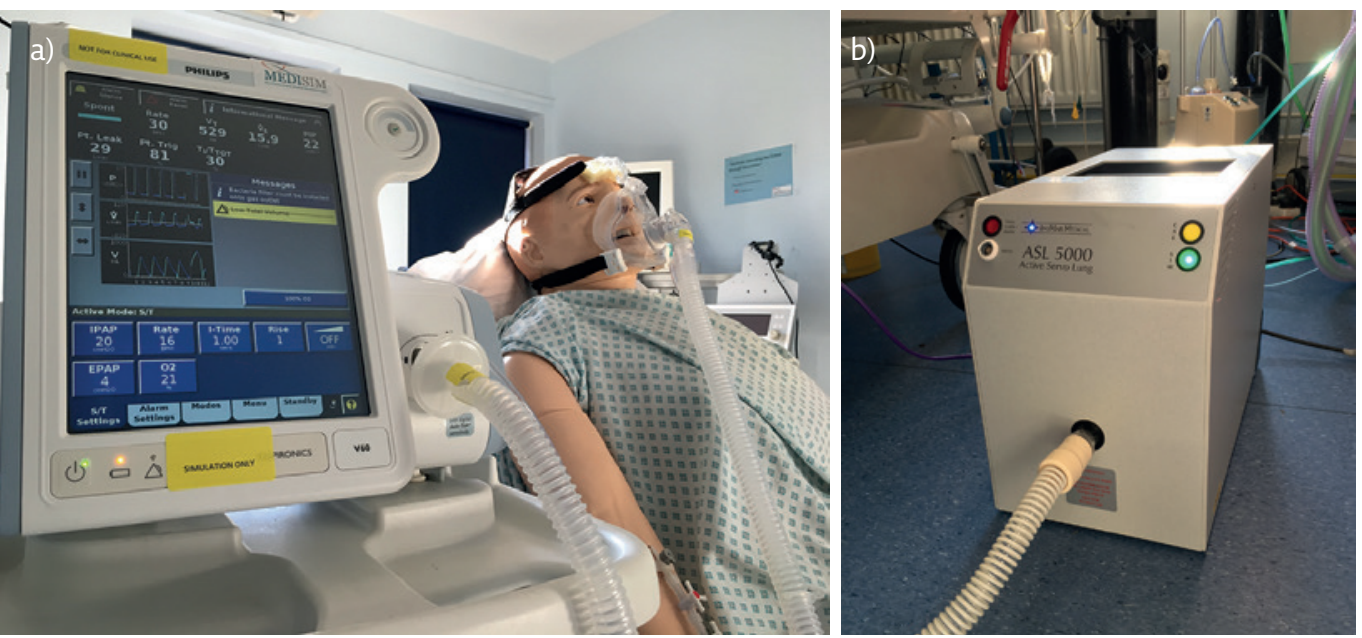

Figure 1 a) SimMan 3G receiving NIV. b) The ASL 5000 breathing simulator. 
resistance, in order to drop the tidal volumes achieved. There will be reduced expansion and decreased breath sounds on auscultation of the affected lung. In addition to this, the patient decompensates from a cardiovascular perspective, becoming hypotensive and tachycardic. The scenario ends when the candidate decompresses the pneumothorax.

Our second scenario, developed for use in our intensive care in situ simulation programme, focuses on NIV optimisation for a patient with an exacerbation of COPD. This scenario begins with the NIV already in place, and the effort of the breathing simulator set to ensure adequate triggering of the ventilator. The ventilator is set to deliver bilevel positive airway pressure with relatively low support (inspiratory positive airway pressure $10 \mathrm{cmH}_{2} \mathrm{O}$, expiratory positive airway pressure $4 \mathrm{cmH}_{2} \mathrm{O}$ ). The resistance is set to achieve the desired tidal volume of ventilation (e.g. $350 \mathrm{~mL}^{-1}$ ). As the scenario progresses, increasing the resistance settings on the breathing simulator will result in a fall in the tidal volume on the ventilator. Inadequate ventilation can be reinforced by provision of an arterial blood gas result, which shows a rising partial pressure of carbon dioxide unless the candidate has appropriately increased the level of support delivered by the ventilator. The candidate must increase the inspiratory positive airway pressure applied by the ventilator and consider intubating the patient to allow invasive ventilation. Alternatively, the patient may begin to tire, which can be simulated by decreasing the effort setting on the breathing simulator, resulting in the failure to trigger support breaths and forcing the machine to deliver "back up" breaths. The scenario ends when the candidate has successfully managed to intubate the patient.

The final scenario we describe here explores the consideration of palliation of a patient receiving NIV. This scenario was aimed for medical doctors about to step up to the registrar tier. The scenario involves a frail patient with a history of frequent admissions for exacerbations of COPD, who is deteriorating despite increasing NIV requirements and is not a candidate for intubation. As the patient deteriorates the effort created by the lung is progressively reduced by the operator, which will eventually stop the patient triggering the ventilator. Again, the candidate should recognise this, as the ventilator will show that "back up" breaths have been initiated. As the patient is not suitable for intubation, the candidate must consider palliation. The focus of this scenario is how the candidate handles discussion with the patient and relative and then sensitively develops an on-going plan for the NIV and end-of-life care.

Other highly realistic scenarios can be developed using breathing simulator technology. We have also created scenarios for use in intensive care, simulating issues such as patient-ventilator dyssynchrony, air trapping and difficulty weaning

\section{Self-assessment questions}

1. The ASL 5000 breathing simulator can be used to realistically simulate:
a) Increased lung resistance seen in severe asthma/COPD
b) A tension pneumothorax
c) Ventilator dyssynchrony
d) Air trapping
e) All of the above

2. Simulation-based learning in NIV has not been demonstrated to:
a) Improve knowledge
b) Improve confidence
c) Improve skills acquisition
d) Improve patient outcomes
e) Improve understanding

3. Which of the following is true with regards simulation-based education in NIV:

a) Lack of appropriate training reduces the efficacy of NIV in the treatment of hypercapnic respiratory failure

b) All medical and nursing staff feel confident to deliver NIV according to guidelines

c) Conferences and lectures are as effective in terms of changing clinical practice

d) Debriefing is of little importance

e) Computer-based simulation is as effective as face-to-face manikinbased simulation

4. Which of the following are not cited as challenges in the administration of simulation-based education:
a) Social distancing requirements
b) Cost
c) Weak evidence base
d) Large number of candidates
e) Adequately trained faculty

5. Breathing simulators can be used to:
a) Improve realism in simulation-based education
b) Replicate a vast number of disease states
c) Teach both noninvasive and mechanical ventilation to multidisciplinary staff
d) Test ventilators, drug delivery and other respiratory equipment
e) All of the above

6. Enhanced realism in simulation may not improve:
a) Candidate engagement
b) Candidate motivation
c) Candidate learning outcomes
d) Candidate performance
e) Candidate stress levels

from ventilatory support. The ASL 5000 breathing simulator can also be effectively used to familiarise intensive care staff with the practicalities of managing different models of ventilator and the multitude of ventilatory modes they provide. This has been of particular importance during the coronavirus disease 2019 (COVID-19) pandemic, where many staff previously unfamiliar with critical care equipment have needed to be 
promptly trained to use ventilators they are inexperienced with.

This breathing simulator and the scenarios described can be robustly used to assess user's understanding of the requirements for NIV, ability to initiate therapy, competence in establishing ventilator settings and ability to respond to the effect of the ventilator on patient pathophysiology. These simulation models are run twice a year as part of a "Registrar Ready" training course for doctors before they enter higher training. The scenarios are also run monthly on the intensive care unit for the in situ training of all multidisciplinary team members. The ability to use the breathing simulator in the hospital setting allows staff to train in their real working environment. In situ simulation further enhances realism, is an excellent way to uncover latent system errors and has been shown to improve patient outcomes [5].

As has been demonstrated with similar simulation-based NIV courses [3], all "Registrar Ready" candidates have historically reported significantly improved self-assessed knowledge, skills and competence levels after completing training. The duration and number of simulation sessions required for any given individual to gain accreditation in using NIV will depend on the candidate's prior experience. Further research is required to determine for how long training gains are retained and when re-training would be required.

In order to maximise the benefits of our scenarios, post-simulation debriefing plays a key role. Effective post-simulation debriefing is key for experiential learning in simulation-based medical education. Active participation, discussion and facilitated reflection of participant's thoughts and actions promotes learning outcomes and enhances future clinical performance.
Unfortunately, delivering simulation-based education can be an expensive, timely and labourintensive resource. The administration of effective simulation-based education has become even more challenging in a world where face-to-face teaching may be restricted due to the social distancing requirements of the COVID-19 pandemic. Online, computer-based systems can also be used for training in NIV, with the benefit of reduced cost, social distancing and increased reach of content [6]. However, conferences, lectures and distanced learning often have little impact in changing clinical practice when compared with practical hands-on training [3]. In a recent randomised control trial comparing computer-based and manikin-based approaches for training in mechanical ventilation, those taught using the manikin scored significantly higher in a final assessment [7]. The immersive, engaging, experiential learning that face-to-face simulation-based education affords is proposed to instil longer lasting behavioural change, knowledge retention and skills acquisition.

\section{Conclusion}

In conclusion, we have found the addition of the ASL breathing simulator to SimMan 36 a great benefit for creating intricate and realistic scenarios that establish training opportunities that would otherwise be a challenge to find. Further work should look at how candidates feel this improves their learning and if such training translates to improved management of NIV on the ward. While this may provide a starting point for use of the ASL breathing simulator with SimMan 3G, there are a multitude of scenarios in which this technology could be used to improve learning and we would encourage you to experiment to find what best works for you.

\title{
Affiliations \\ Daniel Turner, Graham Picton, Elizabeth Harrod, Michele Bossy \\ Medisim, Royal Surrey NHS Foundation Trust, Guildford, Surrey, UK.
}

\section{Supplementary material}

This article has supplementary material available from breathe.ersjournals.com

\begin{abstract}
Authors' contributions
D. Turner and G. Picton wrote the initial manuscript draft. D. Turner and E. Harrod designed, filmed and edited the additional movie file. E. Harrod and M. Bossy contributed to manuscript revisions. All authors read and approved the final manuscript.
\end{abstract}

\section{Conflict of interest}

None declared. 


\section{References}

1. Davidson C, Banham S, Elliott M, et al. British Thoracic Society/Intensive Care Society Guideline for the ventilatory management of acute hypercapnic respiratory failure in adults. BMJ Open Respir Res 2016; 3: e000133.

2. Karim HMR, Burns, KEA, Ciobanu, LD, et al. Noninvasive ventilation: education and training. A narrative analysis and an international consensus document. Adv Respir Med 2019; 87: $36-45$

3. Plumb JOM, Juszczyszyn M, Mabeza G. Non-invasive ventilation (NIV) a study of junior doctor competence. Open Med Educ J 2010; 3: 11-17.

4. Choi W, Dyens O, Chan T, et al. Engagement and learning in simulation: recommendations of the Simnovate Engaged
Learning Domain Group. BMJ Simul Technol Enhanc Learn 2017; 3: 23-32.

5. Goldshtein D, Krensky C, Doshi S, et al. In situ simulation and its effects on patient outcomes: a systematic review. BMJ Simul Technol Enhanc Learn 2020; 6: 3-9.

6. Chatwin M, Hare A, Kurosinski P, et al. Evaluation of the educational outcomes of simulation-based training (SBT) for NIV. Eur Respir J 2015; 46: OA4778.

7. Spadaro S, Karbing DS, Fogagnolo A, et al. Simulation training for residents focused on mechanical ventilation: a randomised trial using mannequin-based versus computer-based simulation. Simul Healthc 2017; 12: 349-355.

\section{Suggested answers}

1. e.

2. d.

3. a.

4. c.

5. e.

6. e. 